\title{
ON SURFACES ENVELOPED BY SPHERES BELONGING TO A LINEAR SPHERICAL COMPLEX*
}

BY

\author{
PERCEY F. SMITH
}

\section{Introduction.}

Surfaces enveloped by spheres cutting orthogonally a fixed sphere were first studied by MouTARD $\dagger$ in the paper: Sur la transformation par rayons vecteurs réciproques, Nouvelles Annales de Mathématiques, ser. 2, vol. 3, 1864. In the discussion there given, the transformation of space named in the title is all important. The volume of DARBOUx, Sur une class remarquable de courbes et de surfaces, 1873 , treats the surfaces and curves of MoutaRD more at length, with especial reference to the case of order 4, the well-known cyclides and bicircular quartics. These surfaces belong to the general class to be studied in this paper, viz. : Surfaces enveloped by spheres belonging to a linear spherical complex.

The configuration of $\infty^{3}$ spheres, the Kugelcomplex, owes its origin to Sophus LiE. $\ddagger$ In the surfaces of MoutaRd the complex involved consists of all spheres intersecting a fixed sphere orthogonally. Other special cases are:

$1^{\circ}$. Complex of all spheres of constant radius. The surface is now either a parallel or tubular surface.

$2^{\circ}$. Complex of all spheres cutting a fixed plane under constant angle. This case has been treated in a paper by the author, On a transformation of Laguerre, Annals of Mathematics, ser. 2, vol. 1, July, 1900.

As in the investigations of Moutard and DaRboux the familiar transformation, inversion in a sphere, serves as the main instrument, so in the following pages the properties of a more general contact-transformation under which spheres remain spheres, suffice for the derivation of the principal theorems.

\footnotetext{
* Presented to the Society June 29, 1900, at the New York summer meeting under the title: Surfaces sibi-reciprocal under contact transformations which transform spheres into spheres. Received for publication September 10, 1900.

$†$ These surfaces MouTARD termed surfaces anallagmatiques.

$\ddagger$ Lie, Ueber Complexe etc., Mathematische Annalen, vol. 5, 1872. The linear spherical complex is the aggregate of all $\infty^{3}$ spheres cutting a fixed fundamental sphere under a constant angle.
}

Trans. Am. Math. Soc. 25 
This transformation depends upon five essential parameters and is an immediate generalization of spherical inversion. The transformation of Laguerre* and spherical inversion appear as equally justified particular cases of this general inversion. The relation of the most general contact transformation under which spheres remain spheres to this general inversion is investigated in $§ 3$.

It is believed that the results obtained in the paper are new, or, at all events, that the point of view has some novelty. In fact, the related investigations to which references are given below employ the line-sphere correspondence of Sophus Lie (loc. cit.), by which theorems in line geometry are translated into theorems on spheres. A direct method has, however, many advantages, for some of the relations of most importance in the geometry of spheres lose their peculiar metric significance when rendered into the language of line geometry; as, e. g., the second theorem of $\S 3$. Furthermore, the duality mentioned hold only for space of three dimensions, and therefore generalization is impossible. The present investigation moves entirely in the realm of the higher spherical geometry. It is to be regarded as analytical, and all equations may immediately be extended to the general case of $n$ dimensions.

Treatment of the general case which shall be of a purely geometrical character is reserved for a later occasion. Means to this end are at hand in the $(1,1)$ correspondence of points in $R_{n+1}$ and oriented spheres in $R_{n}$, the geometrical substratum of which is the so-called minimal projection. $\dagger$ There result in this way theorems of great simplicity and beauty.

In general contact transformations stress must be put upon the notion of orientation. A plane $E$ to which a definite orientation, determined, e. g., by the direction of a normal to that plane, is assigned, may be called oriented. Analytically, such a plane, whose equation in rectangular current coördinates $x, y, z$ is $A x+B y+C z+D=0$, is determined by the five homogeneous coördinates $A, B, C, D, E, \ddagger$ satisfying identically

$$
A^{2}+B^{2}+C^{2}=E^{2} \text {. }
$$

Planes tangent to the imaginary circle at infinity, which we shall call minimum planes, are characterized by $E=0$, i. e., no distinction for orientation exists for such a plane. In fact, the normal to a minimum plane lies in that plane.

The aggregate of a point $P$ and an oriented plane $E$ through $P$ constitutes an oriented surface element, and may be denoted by $(P, E)$. If $P$ is on a surface $F$, and $E$ tangent to $F$ at $P$, then $(P, E)$ is said to belong to $F$.

* Smith, loc. cit.

†CR. LIE, Geometrie der Berïhrungstransformationen, vol. I, p. 453, or KLEIN, Höhere Geometrie, p. 472.

$\ddagger$ With the oriented plane for element of space one has the geometry of reciprocal directions : SмIтH, loc. cit., p. 170. 
Thus a given surface defines two distinct continuous systems each of $\infty^{2}$ oriented surface elements. If, now, $F$ is regarded as defined by one only of these two systems, $F$ is said to be oriented: otherwise, non-oriented. A point $P$ or a curve $C$ likewise defines two systems of oriented surface elements : in the first instance, namely, the $\infty^{2}$ oriented surface elements with the same point $P$, and in the second, the aggregate of the $(P, E)$ 's where $P$ is on $C$ and $E$ a tangent plane to $C$ at $P$. And now we have the definition of tangency fundamental for contact transformations :

Two oriented surfaces, two points or two curves, or a point and oriented surface, point and curve, or curve and oriented surface are tangent if they possess a common oriented surface element.

\section{§1. The linear spherical complex.}

In this section certain known results necessary to the present investigation will be recapitulated.*

In the higher spherical geometry of three dimensional space the element chosen is the oriented sphere. The point and oriented plane are degenerate cases. Analytically, such an element is determined uniquely by the ratios of six coördinates, called hexaspherical coördinates, $x_{1}, x_{2}, \ldots, x_{6}$, which satisfy identically a quadratic relation with non-vanishing discriminant. If the equation of a sphere is written in the form :

$$
x_{5}\left(x^{2}+y^{2}+z^{2}\right)-2 x_{1} x t-2 x_{2} y t-2 x_{3} z t-2 x_{6} t^{2}=0,
$$

$x, y, z, t$ being current rectangular coördinates, and the radius $r$ is made equal to $x_{4} / x_{5}$, the coördinates $x_{i}$ satisfy the simple relation :

$$
\phi(x) \equiv x_{1}^{2}+x_{2}^{2}+x_{3}^{2}-x_{4}^{2}+2 x_{5} x_{6}=0 .
$$

"Tangency" of two elements $x_{i}$ and $y_{i}$ is defined by the vanishing of the polar form :

$$
\phi(x, y) \equiv x_{1} y_{1}+x_{2} y_{2}+x_{3} y_{3}-x_{4} y_{4}+x_{5} y_{6}+x_{6} y_{5} .
$$

If we adopt the convention : the normals to the surface of an oriented sphere are directed outward or inward according as the radius is greater or less than zero, then the condition of tangency becomes that given in the introduction, viz., the possession by the two elements in question, $x_{i}$ and $y_{i}$, of a common oriented surface element. In addition, we deduce :

Two distinct points are tangent if they lie upon a minimum line.

Two oriented planes are tangent if their intersection is a minimum line.

* The dissertation of VIRGIL SNyder, Ueber die linearen Complexe der Lie'schen Kugelgeometrie, Göttingen, 1895, gives a detailed purely geometrical discussion from the standpoint of the linesphere transformation. 
Analytically, the linear spherical complex is defined as the aggregate of the $\infty^{3}$ oriented spheres whose coördinates $x$ satisfy the linear equation

$$
\sum_{i} a_{i} c_{i}=0 \quad(i=1,2, \cdots, 6) .
$$

The geometrical definition appears as follows :

Let $x_{i}$ and $y_{i}$ be two oriented spheres $s$ and $s^{\prime}$ of radii $r$ and $r^{\prime}, d$ the distance of their centers and let the number $\kappa$ be defined by the equation

$$
d^{2}-r^{2}-{r^{\prime}}^{2}+2 r r^{\prime} \kappa=0 .
$$

If $|\kappa|<1, s$ and $s^{\prime}$ intersect under the angle $\cos ^{-1} \kappa$.

In the coördinates the equation just written becomes

$$
x_{1} y_{1}+x_{2} y_{2}+x_{3} y_{3}-\kappa x_{4} y_{4}+x_{5} y_{6}+x_{6} y_{5}=0 .
$$

Comparing equations (1) and (3) we see that the variable sphere $x$ stands in the relation indicated by (2) to the fixed sphere

where

$$
\left(a_{1}, a_{2}, a_{3},-\frac{a_{4}}{\kappa}, a_{6}, a_{5}\right),
$$

$$
\kappa^{2}=\frac{a_{4}^{2}}{\phi(a)+a_{4}^{2}} .
$$

For $|\kappa|<1$, we have the usual statement, viz., the variable sphere intersects the fixed sphere under a constant angle.

We call the fixed sphere the fundamental sphere and $\kappa$ the constant of the complex. The radius $r$ of the fundamental sphere is determined from

$$
r \kappa=-\frac{a_{4}}{a_{6}}, \quad \text { or } \quad r^{2}=\frac{\phi(a)+a_{4}^{2}}{a_{6}^{2}} .
$$

To the complex belong $\infty^{2}$ points, viz., the points of the fundamental sphere, and $\infty^{2}$ oriented planes, viz., the planes enveloping the sphere of radius $r \kappa$ concentric with the fundamental sphere.

The following particular cases are important :

(1) Orthogonal complex, $a_{4}=0$, consisting of all spheres cutting the fundamental sphere orthogonally; here $\kappa=0$. Such a complex will be denoted by $I, I^{\prime}$, etc.

(2) Plane complex, $a_{6}=0$, the fundamental sphere degenerating into an oriented plane. If $r$ is the radius of any sphere of the complex and $p$ the perpendicular distance of its center from the fundamental plane, then the constant $\kappa=p / r$. The fundamental plane has for its equation :

$$
a_{1} x+a_{2} y+a_{3} z+a_{5} t=0, *
$$

\footnotetext{
* For a detailed exposition of the properties of the plane complex, cf. SMrTH, loc. cit.
} 
and is therefore at infinity if $a_{1}=a_{2}=a_{3}=0$. The complex is now the aggregate of all oriented spheres of radius $-a_{5} / a_{4}$. A general plane complex will be symbolized by $E$, the special case just mentioned by $D$.

(3) Special complex, $\phi(a)=0$. The $\infty^{3}$ spheres of the complex are tangent to the fundamental sphere $\left(a_{1}, a_{2}, a_{3},-a_{4}, a_{6}, a_{5}\right)$, since $\kappa=1$.

(4) Complex of all points of space, $a_{i}=0, i \neq 4$. This complex is general, since $\phi(a)=-1$. The coördinates of the fundamental sphere vanish and the latter becomes illusory. The symbol $\Omega$ will be used for this complex.

(5) Complex of all oriented planes of space, $a_{i}=0, i \neq 5$. This complex is special, the fundamental sphere being the plane at infinity.

Two complexes $A$ and $B$ are said to be conjugate or in involution when the polar form

$$
\phi(a, b) \equiv a_{1} b_{1}+a_{2} b_{2}+a_{3} b_{3}-a_{4} b_{4}+a_{5} b_{6}+a_{6} b_{5}
$$

vanishes. If $r$ and $r^{\prime}$ are respectively the radii of the fundamental spheres $s$ and $s^{\prime}, d$ the distance of their centers, and $\kappa$ and $\kappa^{\prime}$ the constants of the complexes, the condition for involution is

$$
d^{2}-r^{2}-{r^{\prime}}^{2}+2 r r^{\prime} \kappa \kappa^{\prime}=0 .
$$

If, however, $A$ is a plane complex, this becomes

$$
p+\kappa \kappa^{\prime}=0,
$$

where $p$ is the perpendicular distance from the center of $s^{\prime}$ to the fundamental plane. Finally, if $A$ and $B$ are both plane complexes and $\theta$ the angle between their fundamental planes, the condition of involution is

$$
\cos \theta+\kappa \kappa^{\prime}=0 \text {. }
$$

The following results are easily derived :

Every complex conjugate to the complex of all points is orthogonal.

Every complex conjugate to the complex of all planes is a plane complex.

If one of two complexes in involution is orthogonal, the fundamental spheres intersect orthogonally.

If one of two complexes in involution is special, its fundamental sphere belongs to the other complex.

Consider now five orthogonal complexes $I_{k}, k=1,2, \ldots, 5$, mutually in involution. Since the complex of all points $\Omega$ is conjugate to each of these, the six complexes $\Omega, I_{k}$, constitute a system of mutually conjugate complexes. Systems of six general complexes mutually conjugate may be constructed.

A degenerate system arises when one of the complexes is special. In this case the special complex is to be reckoned double and its fundamental sphere belongs to each of the remaining four. An interesting case occurs if this special 
complex is the complex of all planes of space, for then the other four complexes are plane complexes.*

§2. Inversion in a spherical complex: definition and properties.

By a linear transformation

$$
\rho A_{i}^{\prime}=\sum_{k} a_{i k} A_{k} \quad(i, k=1,2, \cdots, 6),
$$

the spherical complex $A$ transforms into the complex $A^{\prime}$. We shall consider only such transformations as carry a special complex over into a special complex, i. e., the $a_{i k}$ are assumed such that

$$
\phi\left(A^{\prime}\right) \equiv \lambda \phi(A) \text {. }
$$

Involution is now an invariantive property of the transformation, and the latter depends upon fifteen essential parameters. When $A$ is special, the $A_{i}$ are related to the coördinates $x_{i}$ of the fundamental sphere by the ratios

$$
A_{1}: A_{2}: A_{3}:-A_{4}: A_{6}: A_{5}:: x_{1}: x_{2}: x_{3}: x_{4}: x_{5}: x_{6} \text {. }
$$

With these substitutions and correspondingly for $A_{i}^{\prime}$, the transformation in question becomes a contact transformation under which spheres remain spheres.

Consider in particular the transformation of five essential parameters

defined by

$$
a_{1}: a_{2}: a_{3}: a_{4}: a_{5}: a_{6}, \quad(\phi(a)+0),
$$

$$
A_{i}^{\prime}=A_{i}-\frac{2 \phi(A, a)}{\phi(a)} a_{i} \cdot \dagger
$$

Then $\phi\left(A^{\prime}, a\right)+\phi(A, a)=0$, and by the substitutions $(4), \phi(A, a)$ becomes $\sum_{k} a_{k} x_{k},(k=1,2, \ldots, 6)$. The transformation (5) has, therefore, the following properties :

$A$ sphere belonging to the complex $\sum_{\kappa} a_{\kappa} x_{\kappa}=0$ is invariant; every sphere of this complex which is tangent to an arbitrary sphere $x$ is tangent also to the transformed sphere $x^{\prime}$, and reciprocally.

These theorems furnish a purely geometrical definition of the transformation.

The transformation is uniquely determined by the complex $a$, and conversely. We shall call the transformation inversion in the linear spherical complex $a$;

The fundamental sphere of $a$ is the fundamental sphere of the transformation, and its center the center of the transformation. We shall indicate the inversion in the complex $a$ by $(a)$.

* SMITH, loc. cit., p. 165.

$\dagger$ The determination of (5) is simply this: $A_{i}^{\prime}$ shall have the form $\lambda A_{i}+\mu a_{i}$.

$\ddagger$ Cf. the memoir of E. MüLLER, Ueber die Geometrie orientirter Kugeln etc., Monatshefte für Mathematik und Physik, 1898, p. 314. Under the line-sphere transformation this corresponds to the dualistic inversion in a line complex. 
Particular cases are the following:

When $a$ is an orthogonal complex, $(a)$ is the well known spherical inversion in the fundamental sphere.

When $a$ is a plane complex, $(a)$ is the transformation by reciprocal directions of Lagiuerre.*

When $a$ is a $D$, say $\left(0,0,0,1, \frac{1}{2} c ; 0\right)$, corresponding spheres under $(a)$ are concentric and their radii $r$ and $r^{\prime}$ satisfy the relation $r^{\prime}+r=-c$, i. e., $(D)$ is a dilatation.

When $a$ is the complex of all points $\Omega,(\Omega)$ merely changes orientation.

The fact of the transformation of the complex $A$ under $(a)$ into $A^{\prime}$ will be indicated by

or, since $(a)$ is involutory,

$$
A(a) A^{\prime},
$$

$$
A^{\prime}(a) A \text {. }
$$

We now establish certain theorems.

Theorem 1. A complex $A$ not a plane complex may be transformed by a unique dilatation $(D)$ into an orthogonal complex $I$.

In fact, by inversion in

$A$ becomes an $I$ :

$$
D:\left(0,0,0, A_{6}, \frac{1}{2} A_{4}, 0\right),
$$

$$
\left(A_{1}, A_{2}, A_{3}, 0, \frac{\phi(A)-A_{1}^{2}-A_{2}^{2}-A_{3}^{2}}{2 A_{6}}, A_{6}\right) .
$$

The spherical inversion $(I)$ has the same center as $(A)$, and its power, i. e., the square of the radius of its fundamental sphere, is $R^{2}\left(1-\kappa^{2}\right)$, where $R$ is the radius of the fundamental sphere and $\kappa$ the constant of $A$. The radius of the spheres of $D$ is $-\frac{1}{2} R \kappa$. If $A$ is orthogonal, then $D$ is $\Omega$, and $I$ and $A$ are identical.

Theorem 2.-If $A(D) I$, then $\Omega(D) D^{\prime}$, where $D^{\prime}$ is $\left(0,0,0, A_{6}, A_{4}, 0\right)$, and $D^{\prime}$ and $A$ are in involution.

This easily follows, since $\Omega$ and $I$ are in involution.

Theorem 3.-Any complex A may be transformed by a spherical inversion $(I)$ into a plane complex, and any pencil into a pencil of plane complexes.

For if $a_{4}=0, A$ becomes by $(a)$ a plane complex if the $a$ 's satisfy

$$
A_{6} \phi(a)-2 \phi(A, a) a_{6}=0 \text {. }
$$

In fact, when the center of $(a)$ is chosen in the common intersection of the fundamental spheres of $A$ and $B$, then every complex $\lambda A+\mu B$ inverts into a plane complex.

* Cf. Smith, loc. cit., p. 157. 
If $A(a) A^{\prime}$, then the inversion $(A)$ is transformed by $(a)$ into $\left(A^{\prime}\right)$. Thus the theorems take the form:

$$
\begin{aligned}
& (A)=(D I D) ; \\
& \left(D^{\prime}\right)=(D \Omega D) ; \\
& (A)=\left(I^{\prime} E I^{\prime}\right) .
\end{aligned}
$$

Hence: The general inversion is uniquely compounded of a dilatation, a spherical inversion, and the same dilatation, or of a spherical inversion, inversion in a plane complex, and the same spherical inversion, the latter resolution not being unique.

Since, now, by an $(I)$, two general complexes $A$ and $A^{\prime}$ may be transformed into plane complexes, $E$ and $E^{\prime}$, then

$$
\left(A A^{\prime}\right)=\left(I E E^{\prime} I\right) \text {. }
$$

Theorems established for the transformation $\left(E E^{\prime}\right)^{*}$ are thus transformed into theorems on the transformation $\left(A A^{\prime}\right)$, and we may state the following results :

Theorem 4.-The transformation of space which is compounded of successive inversions in two spherical complexes may be resolved in $\infty^{1}$ ways into successive inversions in two other spherical complexes. The four complexes belong to the same pencil; and, of the two latter, one may be chosen arbitrarily, and the other is then uniquely determined.

Since in the pencil $\lambda A+\mu A^{\prime}$ there is at least one orthogonal complex $I$ and one plane complex $E$, the transformation $\left(A A^{\prime}\right)$ is identical with a spherical inversion $(I)$ or inversion in a plane complex $(E)$ followed by a second inversion, or an inversion compounded with the same $(I)$ or $(E)$; i. e.,

$$
\begin{aligned}
\left(A A^{\prime}\right) & =(I B)=\left(B^{\prime} I\right) \\
& =(E C)=\left(C^{\prime} E\right) .
\end{aligned}
$$

Theorem 5.-The inversions $(A)$ and $\left(A^{\prime}\right)$ are commutative when, and only when, the complexes $A$ and $A^{\prime}$ are in involution. Then $\left(A A^{\prime}\right)$ is an involutory transformation. $\dagger$

An important case is where $(A)$ and $\left(A^{\prime}\right)$ are commutative and have the same fundamental sphere $\sigma$. One has then

$$
\kappa \kappa^{\prime}=1 \text {. }
$$

\footnotetext{
* Cf. SMrth, loc. cit., p. 169, seq., and the paper by WIENER : Ueber die aus zwei Spiegelungen zusammengesetzten Verwandtschaften, Leipziger Berichte, 1891, p. 660.

It is easy to show that $\left(A A^{\prime}\right)=\left(B B^{\prime}\right)$ when and only when

$$
\frac{\phi\left(A, A^{\prime}\right)}{\sqrt{\phi(A) \phi\left(A^{\prime}\right)}}=\frac{\phi\left(B, B^{\prime}\right)}{\sqrt{\phi(B) \phi\left(B^{\prime}\right)}} .
$$
}


If $(I)$ is the spherical inversion in $\sigma$, then

i. e.,

$$
\left(A A^{\prime}\right)=(I \Omega)=(\Omega I),
$$

$$
(A)=\left(A^{\prime} \Omega I\right)=\left(I \Omega A^{\prime}\right) \text {. }
$$

Inversion in a complex with constant $\kappa$ may therefore be resolved into inversion in a complex with the same fundamental sphere and constant equal to $1 / \kappa$, change in orientation and inversion in that sphere.

Theorem 6.-There are two complexes $C_{1}$ and $C_{2}$ such that for any two inversions $(A)$ and $(B)$

and then

$$
\begin{aligned}
& \left(A C_{1}\right)=\left(C_{1} B\right), \\
& \left(A C_{2}\right)=\left(C_{2} B\right),
\end{aligned}
$$

$$
\left(C_{1} C_{2}\right)=\left(C_{2} C_{1}\right) \text {; }
$$

that is, any two inversions may be transformed into each other by two inversions, and these two inversions are commutative.

For example, the complex $A$ is transformed into $\Omega$ by inversion in

$$
A_{0}:\left(A_{1}, A_{2}, A_{3}, A_{4} \pm \sqrt{-\phi(A)}, A_{5}, A_{6}\right),
$$

i. e., a spherical complex $A$ may be inverted into the complex of all points by two inversions for each of which the fundamental sphere is identical with the fundamental sphere of $A$.

If $\kappa_{0}$ is the constant of $A_{0}$, then $\kappa_{0}^{2}-2 \kappa \kappa_{0}+1=0$. The complexes $A_{0}$ and $A_{0}^{\prime}$ are therefore in involution. In particular, if $A$ is a plane complex, so also is $A_{0}$. Again, by theorems 1 and 2

If, therefore, $S$ is a sphere of $A$ and

$$
\left(A D^{\prime}\right)=\left(D^{\prime} A\right)
$$

then since

$$
S(A) S\left(D^{\prime}\right) S^{\prime}
$$

$$
S\left(D^{\prime}\right) S^{\prime}(A) S^{\prime},
$$

$S^{\prime}$ also belongs to $A$, and the spheres of $A$ are concentric in pairs and inverse with respect to $\left(D^{\prime}\right)$. When $A$ is orthogonal, $S$ and $S^{\prime}$ differ only in orientation.

Theorem 7.-If $\left(A D^{\prime}\right)=\left(D^{\prime} A\right)$ and $(A)=\left(A_{0} \Omega A_{0}\right)$, then by $\left(A_{0}\right),\left(D^{\prime}\right)$ is transformed into a spherical inversion $\left(I^{\prime}\right)$, and the concentric spheres of $A$, viz., $S$ and $S^{\prime}$, invert by $\left(A_{0}\right)$ into two points inverse with respect to $\left(I^{\prime}\right)$.

Finally we may add this theorem : 
Theorem 8.-By a general inversion $\left(A_{0}\right)$ which transforms $(\Omega)$ into $(A)$ the system of six mutually conjugate complexes consisting of $\Omega$ and five orthogonal complexes whose fundamental spheres intersect orthogonally is inverted into a general system of six conjugate complexes of which $A$ is one. In the same way the degenerate system made up of the complex of all planes and four conjugate plane complexes transforms into a general degenerate system.

§3. The group $G_{15}$ of all contact transformations which transform spheres into spheres.

By successive applications of theorem 4 of the preceding section, we have :

$A$ transformation $(T)$ compounded of any number $n$ of inversions may be resolved into one inversion followed by $n-1$ spherical inversions, or $n-1$ spherical inversions followed by an inversion.

If now $n$ is odd, by theorem 5 we may replace the inversion in question so that the number of spherical inversions shall always be odd. But an odd number of spherical inversions reduces to a displacement and one spherical inversion, or to one spherical inversion followed by a displacement; hence the theorem:

The transformation $(T)$ may be reduced to one of the forms

$$
\begin{aligned}
& (A I S)=\left(S^{\prime} I^{\prime} A^{\prime}\right), \\
& (A \Omega I S)=\left(S^{\prime} I^{\prime} \Omega A^{\prime}\right),
\end{aligned}
$$

where $S$ is a displacement. Or, since a displacement is compounded of at the most four symmetries, the transformation $(T)$ may be reduced to successive inversions in six and seven spherical complexes respectively.*

The transformation $(T)$ depends on 15 essential parameters and is the most general contact transformation which transforms spheres into spheres. For, analytically, the most general transformation of this kind is the linear transformation

where

$$
\begin{array}{ll}
\rho x_{i}^{\prime} & =\sum_{k} a_{i k} x_{k} \\
\phi\left(x^{\prime}\right) & \equiv \mathrm{K} \phi(x),
\end{array}
$$

and it is easy to resolve this into successive inversions. The $\infty^{15}$ transformations form two continuous systems, of which $(a)$ and $(b)$ are typical forms. The transformations $(a)$ of themselves form a continuous group, and the entire set of $(a)$ and $(b)$ give the group $G_{15}$ of all contact transformations under which spheres remain spheres.

\footnotetext{
* A transformation of the type (a) may be made to assume the form $\left(\left(A_{1} A_{2}\right)\left(A_{3} A_{4}\right)\left(A_{5} A_{6}\right)\right)$, where $A_{1}$ and $A_{2}, A_{3}$ and $A_{4}, A_{5}$ and $A_{6}$ are in involution. That is, (T) in this case may be resolved into three involutory transformations. Then by the line sphere duality, any collineaeation may be resolved into three skew reflections. Cf. E. B. WiLson, Transactions of the American Mathematical Society, vol. 1, p. 193, 1900.
} 
In the Mathematische Annalen, vol. 5, p. 186, Sophus LiE gives the theorem that every transformation of the type here considered is compounded of dilatations and spherical inversions. Since, by theorem $1,(A)=(D I D)$, the truth of LIE's result is established.

The resolutions $(a)$ and $(b)$ are, it is believed, new, and show that what is essentially novel in such a transformation can be learned by the study of the inversion in one general complex.

\section{§4. Surface sibi-reciprocal under a general inversion.}

In this section the problem under discussion is attacked.

- The transformation of a surface $F$ into a surface $F^{\prime}$ by $(A)$ is effected as follows: Let $(P, E)$ be an oriented surface element of $F$, and let $P$ and $E$ by $(A)$ become the oriented spheres $s$ and $s^{\prime}$; then $s$ and $s^{\prime}$ have a common oriented surface element $\left(P^{\prime}, E^{\prime}\right)$, and this belongs to $F^{\prime}$. We note that $F^{\prime}$ is necessarily an oriented surface. If then $F$ under $(A)$ transforms into itself, $F$ must in general be oriented. In particular : a non-oriented surface is sibireciprocal under $(\Omega)$, and conversely, if

then $F$ is non-oriented.

$$
F(\Omega) F
$$

Let now $F^{\prime}(A) F$, and consider any sphere $s$ of $A$ tangent to $F$; then since $s(A) s, s$ must touch $F$ again; i. e., the surface $F$ is the envelope of spheres belonging to $A$.

It follows from theorem 6 of $\S 2$, that if $\left(A_{0}\right)=(A \Omega A)$ and $F\left(A_{\mathrm{Q}}\right) f$, then $f(\Omega) f$, and $f$ is an ordinary point locus. The result is

Theorem 1.-A surface enveloped by spheres belonging to a linear spherical complex may always be derived by a general inversion from a primitive curve or surface regarded as a point locus.

This theorem furnishes the point of view for the following. If the primitive is a curve, the derived surface is an annular surface, one system of lines of curvature being circles.

We now establish various theorems by the aid of the results of $\S 2$.

By theorem 1 of $\S 2$, if $\left(A_{0}\right)=\left(D_{0} I_{0} D_{0}\right)$, then let, since $f\left(A_{0}\right) F$,

$$
f\left(D_{0}\right) f^{\prime}\left(I_{0}\right) f^{\prime \prime}\left(D_{0}\right) F
$$

i. e., the surface and its primitive are transformed by the same dilatation $\left(D_{0}\right)$ into surfaces inverse under a spherical inversion concentric with $(A)$.

From the known properties of dilatations * and spherical inversions, we deduce the following theorems, under the assumption that the center of inversion has general position with respect to the primitive :

* Cf., e. g., the paper by S. RoBerTs, Proceedings of the London Mathematical Society, 1873, p. 94. 
If the primitive is a surface of order $m$, class $n$, and if $2 N$ is the order of its non-singular minimum developable,* then the derived surface is of order and class $2(2 m-n+2 N)$ and passes $2 m-n+2 N$ times through the imaginary circle at infinity.

If the primitive is a curve of order $m$, rank $r$, passing $2 p$ times through the imaginary circle at infinity, then the derived annular surface is of order and class $2(2 m+r-4 p)$ and passes $2 m+r-4 p$ times through the imaginary circle at infinity.

From these results we may state

Theorem 2.-In general, if a surface is enveloped by spheres belonging to a linear spherical complex, the order and class are equal, the surface intersects the plane at infinity along the imaginary circle, and the minimum developable is singular.

The points of the fundamental sphere being unchanged by $\left(A_{0}\right)$, we have

Theorem 3.-The curve of intersection of the primitive surface and the fundamental sphere is a double line on the derived surface. The points of intersection of the primitive curve and fundamental sphere are conical points of the derived annular surface. $\dagger$

We may now obtain another theorem from the resolution of $\left(A_{0}\right)$ (theorem $3, \S 2)$ :

$$
\left(A_{0}\right)=\left(I_{0}^{\prime} E_{0} I_{0}^{\prime}\right)
$$

Let $f\left(I_{0}^{\prime}\right) f^{\prime}$, and $F\left(I_{0}^{\prime}\right) F_{0}^{\prime}$; then since $f\left(A_{0}\right) F$, we must have

$$
f^{\prime}\left(E_{0}\right) F_{0}^{\prime} \text {. }
$$

From the properties of the inversion $\left(E_{0}\right)$, if the point $P$ on $f^{\prime}$ transform by $\left(E_{0}\right)$ into the oriented sphere $s$ touching $F_{0}^{\prime}$ in $P^{\prime}$ and $P^{\prime \prime}$, then the circle passing through $P, P^{\prime}$ and $P^{\prime \prime}$ intersects $f^{\prime}$ and $F_{0}^{\prime}$ orthogonally, and its center lies in the fundamental plane of $E_{0} \cdot \frac{+}{+}$ Transforming back by $\left(I_{0}\right)$ we get

Theorem 4.-If the point $M$ on $f$ invert by $\left(A_{0}\right)$ into the sphere $S$, then the circle drawn through $M$ orthogonal to $f$ and the fundamental sphere of $\left(A_{0}\right)$ intersects $S$ and $F$ orthogonally in the points of contact of $S$ and $F \cdot \S$

The results just deduced are based upon the fact that a linear spherical complex $A$ not special may be transformed by inversion in a complex $A_{0}$ into the

\footnotetext{
* The singular minimum developable of a surface containing the imaginary circle at infinity is the developable formed by planes tangent to the surface along this line. If $f$ has no minimum developable except the singular developable, as is the case for the sphere, then $N=0$.

$\dagger$ Interesting exceptions to the theorems 2 and 3 are noted below in 88 .

$\ddagger$ SMITH, loc. cit., p. 161, or DARBoux, Leçons sur la théorie générale des surfaces, vol. I, p. 261.

$\S$ Thus by the general theorem of Ribaucour (cf. DaRboux, loc. cit., vol. II, p. 330), the primitive surface $f$ and the derived $F$ belong to the same cyclic system, which may be obtained from $f$ by inversions in the fundamental sphere with varying constant $\kappa$.
} 
complex of all points $\Omega$. We now write the equations of the transformation, using the defining formulas $(5)$ of $\S 2$. If the origin is taken for the center of the inversion, then $a_{1}=a_{2}=a_{3}=0$.

Denoting the radius of the fundamental sphere by $r_{0}$ and the constant of $A_{0}$ by $\kappa_{0}$, the point $(x, y, z)$ inverts into the oriented sphere with center $\left(x^{\prime}, y^{\prime}, z^{\prime}\right)$ and radius $r^{\prime}$ such that

$$
\begin{cases}x^{\prime}=\frac{s x}{x^{2}+y^{2}+z^{2}-d^{2}}, & y^{\prime}=\frac{s y}{x^{2}+y^{2}+z^{2}-d^{2}}, \\ z^{\prime}=\frac{s z}{x^{2}+y^{2}+z^{2}-d^{2}}, & r^{\prime}=\frac{d\left[r_{0}^{2}-\left(x^{2}+y^{2}+z^{2}\right)\right]}{x^{2}+y^{2}+z^{2}-d^{2}},\end{cases}
$$

where $* d=-r_{0} \kappa_{0}, s=r_{0}^{2}\left(1-\kappa_{0}^{2}\right)$.

These equations show that the $\infty^{2}$ points of the sphere $x^{2}+y^{2}+z^{2}=d^{2}$ correspond to the $\infty^{2}$ oriented planes of the complex $A$. We have already seen that such planes touch a sphere concentric with the fundamental sphere. Points of the primitive, therefore, lying on the sphere $x^{2}+y^{2}+z^{2}=d^{2}$ go over into bitangent or singular tangent planes to the derived surface.

These considerations give

Theorem 5.-If a surface is enveloped by $\infty^{2}$ spheres belonging to a linear spherical complex $A$, then a developable, whose planes belong to $A$ and touch a sphere concentric with its fundamental sphere, has double contact with that surface.

The line joining a point on the sphere $x^{2}+y^{2}+z^{2}=d^{2}$ and the center is normal to the corresponding plane. In fact, the curve of intersection of this sphere with the primitive surface and the developable of bitangent planes are reciprocal under a polar reciprocation in the center. The modification of these results for an annular surface is obvious.

Let now the primitive be a surface of order $m$ passing $r$ times through the imaginary circle at infinity. Then the derived surface contains a double curve (theorem 3) of order $2(m-r)$ lying on the fundamental sphere, while the developable having double contact with the surface (theorem 5) is the polar reciprocal of a curve of the same order. If the primitive is a curve of order $m$ passing $2 p$ times through the imaginary circle, then the derived annular surface possesses $2(m-p)$ conical points and the same number of singular tangent planes.

We pass now to the consideration of the locus of centers of the spheres enveloping the surface under discussion. The equations (6) give the point trans-

* Thus is established a $(1,1)$ involutory correspondence between the points of space and spheres of a linear spherical complex. The study of this correspondence is reserved for a later occasion. Geometrically, the point is a limiting point of the coaxial system determined by the corresponding sphere and the fundamental sphere of the complex. 
formation of the primitive into this locus.* This is a $(1,2)$ correspondence, a point $\left(x^{\prime}, y^{\prime}, z^{\prime}\right)$ yielding two points $(x, y, z)$ inverse under a spherical inversion $\left(I^{\prime}\right)$ on the origin (theorem $7, \S 2$ ).

In general, then, the transformation of the primitive into the locus of centers is birational. $\dagger$ Corresponding points lie on a line through the origin and the imaginary circle at infinity is the "fundamental curve." We easily derive

Theorem 6.-If the primitive is a surface of order $m$, class $n$, passing $r$ times through the imaginary circle at infinity, the derived surface is enveloped by $\infty^{2}$ spheres whose centers lie upon a surface of order $2(m-r)$, class $2 m+n-4 r$, having at the center of inversion a conical point of order $m-2 r$.

If the primitive is a curve of order $m$ passing $2 p$ times through the imaginary circle at infinity, the derived annular surface is enveloped by $\infty^{1}$ spheres whose centers lie upon a curve of order $2(m-p)$ passing $m-2 p$ times through the center of inversion.

Let $F$ denote the surface under discussion, i. e., $F(A) F$. Since by theorem $1, \S 2$, we have $(A)=(D I D)$, if $F(D) F^{\prime \prime}$, then necessarily $F^{\prime}(I) F^{\prime}$, or the surface $F$ is transformed by a dilatation into a surface $F^{\prime}$ sibi-reciprocal under a spherical inversion on the center of $A$, and having the same locus of centers. $\ddagger$ Let us call this locus of centers $F_{0}$, and the center of inversion $O$.

The points of contact of $S$, a sphere of $A$, with $F$ are now easily constructed : viz., let $E$ be a tangent plane to $F_{0}$ at $P$, the center of $S$, and let $S^{\prime}$ be the sphere of $I$ with center $P$. Then $S^{\prime}$ touches $F^{\prime}$ in the points of intersection of $S^{\prime}$ and a line drawn through $O$ perpendicular to $E$. The points of contact of $S$ with $F$ lie on the lines joining these points on $S^{\prime}$ with $P$. (Cf. theorem 4.) If, now, $E$ is a minimum plane, then $S^{\prime}$ : touches $F^{\prime}$ on the imaginary circle at infinity, and the same is true of $S$ and $F$. Hence

Theorem 7.-The minimum developable of the locus of centers is the singular minimum developable of the surface $F$.

\$5. The general inversion as a transformation of oriented surface elements.

If the equation of a plane be written in current homogeneous rectangular coordinates $x_{1}, x_{2}, x_{3}, x_{4} \S$ in the form

$$
(u x) \equiv u_{1} x_{1}+u_{2} x_{2}+u_{3} x_{3}+u_{4} x_{4}=0,
$$

* This transformation for the case $s=2 r_{0}^{2}, d^{2}=-r_{0}^{2}$ is given by DARBoux, Sur une classe remarquable etc., p. 123 , and loc. cit., vol. III, p. 493, and appears below, $\& 8$.

$\dagger$ Exception discussed below, \& 8 .

$\ddagger$ Cf. DARBoux, Sur une classe etc., p. 255.

$\S$ The plane at infinity is supposed to be $x_{4}=0$. 
and if $u_{0}$ be defined by $u_{0}^{2}=u_{1}^{2}+u_{2}^{2}+u_{3}^{2}$, then the ratios

$$
x_{1}: x_{2}: x_{3}: x_{4}, \quad u_{0}: u_{1}: u_{2}: u_{3}: u_{4}
$$

will determine a unique oriented surface element $(x, u)$.

The construction given at the end of the previous section for the surface element corresponding to a given surface element $(P, E)$ enables us to deduce the equations of inversion in the complex $A_{0}$ in terms of the above coördinates. Taking the origin for center of inversion, $\kappa_{0}$ for the constant of $A_{0}, r_{0}$ for the radius of the fundamental sphere, we easily derive for the transformation of $(x, u)$ into $\left(x^{\prime}, u^{\prime}\right)$ by $\left(A_{0}\right)$ the following equations :

where

$$
\left\{\begin{array}{l}
\rho x_{i}^{\prime}=x_{i} \psi-\left(x_{i} u_{0}-x_{4} u_{i} d\right)\left(U-r_{0}^{2} x_{4}^{2}\right) \\
\rho x_{4}^{\prime}=x_{4} \psi \\
\sigma u_{0}^{\prime}=\psi \\
\sigma u_{i}^{\prime}=u_{i}\left(U-d^{2} x_{4}^{2}\right)+2 x_{i} x_{4}\left(u_{4}+d u_{0}\right) \\
\sigma u_{4}^{\prime}=u_{4}\left(U-d^{2} x_{4}^{2}\right)-\left(U+r_{0}^{2} x_{4}^{2}\right)\left(u_{4}+d u_{0}\right), \quad(i=1,2,3),
\end{array} \quad(i=1,2,3),\right.
$$

$$
\begin{aligned}
\psi & \equiv u_{0}\left(U+d^{2} x_{4}^{2}\right)+2 u_{4} d x_{4}^{2}, \\
U & \equiv x_{1}^{2}+x_{2}^{2}+x_{3}^{2}, \\
d & \equiv-r_{0} \kappa_{0},
\end{aligned}
$$

and $\rho$ and $\sigma$ are factors of proportionality. From these, ${ }^{*}$ we get

$$
\begin{aligned}
\rho \sigma\left(u^{\prime} x^{\prime}\right) & \equiv\left(U-d^{2} x_{4}^{2}\right) \psi(u x), \\
\rho \sigma\left(u^{\prime} d x^{\prime}\right) & \equiv\left(U-d^{2} x_{4}\right) \psi(u d x),
\end{aligned}
$$

$(u d x)$ being the linear differential expression $u_{1} d x_{1}+u_{2} d x_{2}+u_{3} d x_{3}+u_{4} d x_{4}$; these identities show the transformation to be a contact transformation. Assuming $\rho=\sigma=\psi$, we may readily verify the involutory property. If $d=0$, the transformation is a point transformation, the usual spherical inversion.

Without entering into details, we may state the following theorems, it being understood that the complex of all points $\Omega$ inverts by $\left(A_{0}\right)$ into the complex $A$.†

The $\infty^{1}$ minimum surface elements along a minimum line intersecting the fundamental sphere in $P$ give the same element $(P, E)$, whose plane $E$ belongs to $A$.

* In the equations just written, the coördinates $x, u$ were chosen rather than the customary $x, y, z, p, q$, for homogeneity and to emphasize orientation.

†The important thing is this: The $\infty^{2}$ minimum planes constitute the linear congruence common to the complexes of all points and all planes. By $\left(A_{0}\right)$, this congruence becomes the congruence (special) common to $A$ and the special complex whose fundamental sphere is $U=d^{2} x^{2}$. 
An element of the imaginary circle at infinity gives the $\infty^{2}$ elements of a sphere of $A$, belonging to $\psi=0$; this sphere is of radius $d$ and its center is on the asymptotic cone of the fundamental sphere.

An element of the sphere $U=d^{2} x_{4}^{2}$ transforms into $\infty^{1}$ parallel elements with common point on the imaginary circle at infinity.

The sphere $U=d^{2} x_{4}^{2}$ and the imaginary circle at infinity are "fundamental configurations" for the transformation.

Consider, now, the curve of intersection $c$ of the fundamental sphere and the non-singular minimum developable circumscribing the primitive. The $\infty^{1}$ elements common to this developable and the primitive invert into elements of the derived surface along the curve $c$. Since the planes of the elements belong to $A$, we have the

THEOREM.-The intersection of the fundamental sphere and the non-singular minimum developable circumscribing the primitive is a line of curvature on the derived surface along which the principal spheres have four-point contact.

The double line of theorem $3, \S 4$, and the curve just mentioned constitute the entire intersection of the derived surface and the fundamental sphere. It is otherwise obvious that any line common to the fundamental sphere and the derived surface which is not a multiple line on that surface must be a line of curvature, since its elements must remain unchanged under the inversion $(A)$.

The theorem enunciated is merely a case of the well-known general theorem :

The $\infty^{1}$ surface elements of the primitive surface along a line of curvature invert into $\infty^{1}$ elements of the derived surface along a line of curvature.

For the curve of contact of the primitive surface with the circumscribed minimum developable is a singular line of curvature. $*$

Let the elements $(P, E)$ and $\left(P^{\prime}, E^{\prime}\right)$ be such that $P$ and $P^{\prime}$ lie on a minimum line while $E$ and $E^{\prime}$ intersect in that line. Then the transformed elements will have the same property, whence the theorem :

The two continuous systems of oriented elements of the primitive along a minimum line in that surface invert into elements of the derived surface along two distinct minimum lines intersecting on the fundamental sphere and lying in a plane belonging to $A$.

§6. The surface of singularities of the general quadratic spherical complex.

Particular interest attaches to the case when the primitive surface $f$ of the preceding sections is a cyclide, i. e., a surface of the fourth order containing the imaginary circle at infinity as double line. This surface is sibi-reciprocal under

* Cf. Darboux, Sur une classe remarquable etc., page 11. Also Snyder, American Jour nal of Mathematics, vol. 22, p. 97, 1900. 
five commutative spherical inversions, and being non-oriented, under $(\Omega)$ as well. The class of the cyclide is 12 , and the non-singular minimum developable is of order 16. * By a general inversion in a complex having no special relation to the cyclide this surface is transformed into the surface of singularities of the general quadratic spherical complex. $\dagger$

We have then the following theorems for this surface:

The surface of singularities of the general quadratic spherical complex is sibi-reciprocal under six commutative general inversions $\left(A_{i}\right)$. With each $\left(A_{i}\right)$ is associated a cyclide $f_{i}$ into which the surface transforms by an inversion $\left(A_{i}^{\prime}\right)$ concentric with $\left(A_{i}\right)$.

The surface is of order and class 24 , and contains the imaginary circle at infinity as twelve-fold line, and also six double lines, the intersections of $f_{i}$ and the fundamental sphere $\sigma_{i}$ of the corresponding inversion $\left(A_{i}\right)$. These double lines are sphero-quartic curves.

The surface is the envelope in six ways of $\infty^{2}$ spheres belonging to an $A_{i}$ whose centers lie on a Kummer quartic $F_{i} \cdot \frac{+}{+}$ The six quartics $F_{i}$ are confocal, and are inscribed in the minimum developable of the surface of singularities, which is also the singular minimum developable for that surface. Thus the focal lines of the $F_{i}$ are the focal lines of the surface.

The surface is enveloped by six systems of bitangent planes each belonging to a developable of order 8 circumscribed about a sphere concentric with $\left(A_{i}\right)$ along a sphero-quartic. The total intersection of the surface with a fundamental sphere $\sigma_{i}$ is made up of the double line already mentioned, and a curve of order 16, which is a line of curvature of the surface along which the principal spheres have four-point contact. The lines of curvature of the surface are algebraic and of order 32, and are inverted by each of the six inversions $\left(A_{i}^{\prime}\right)$ into the lines of curvature of a cyclide $f_{i}$. The surface contains 32 minimum lines, arranged in six ways in 16 pairs, such that the intersection of each pair is on a fundamental sphere $\sigma_{i}$, while the plane of each pair belongs to $\left(A_{i}\right)$.

Since a cyclide $f_{i}$ depends upon 13 , an inversion $\left(A_{i}\right)$ upon 5 essential constants, we have, finally,

The surface of singularities of the general quadratic spherical complex depends upon 18 essential constants.

These results are believed to be new.

* Cf. Darboux, Sur une classe remarquable de courbes et de surfaces, p. 305.

† LIE, loc. cit., p. 178. Cf. also SNYDER, Bulletin of the Amerioan Mathematical Society, vol. 4, p. 152.

+ i. e., a quartic containing a double conic.

$\S$ Cf., however, SNyder, Bulletin of the American Mathematical Society, May, 1900. 


\section{§ 7. Cyclide of Dupin.}

To illustrate the preceding theorems when the surface is annular, we may consider the case when the primitive is a right line (or circle). Then the derived surface is enveloped by $\infty^{1}$ spheres belonging to the complex $A$, whose centers lie upon a conic, $i$. e., is a cyclide of Dupin, of order and class 4 , containing the imaginary circle two-fold. The two pencils of oriented planes containing the primitive invert into the spheres of the second generation.

Space does not permit us to carry the discussion further. This relation of the cyclide of Dupin to the right line is believed to be new, and the discussion of the properties of the surface from this point of view is of great simplicity and interest.

\section{§ 8. Cases of exception to the general theorems.}

In the statement of theorem $6, \S 4$, the assumption was made that the primitive surface $f$ was not sibi-reciprocal under the spherical inversion $\left(I^{\prime}\right)$. We easily derive the following results, if $f\left(I^{\prime}\right) f$.

If $f\left(I^{\prime}\right) f$, the derived surface $F$ is the complete parallel of a surface $F^{\prime}$, which is sibi-reciprocal under the spherical inversion $(I)$, concentric with $\left(I^{\prime}\right)$.* If $f_{0}$ be the locus of centers of spheres belonging to $\left(I^{\prime}\right)$ and enveloping $f$, then $f_{0}$ and the locus of centers for $F$ are polar reciprocals under a polar reciprocation in the center of inversion with power $\frac{1}{2} s, s$ having the same signification as above $(\S 4)$. Theorems $3,4,5,7(\S 4)$ still hold.

In particular, if the primitive is a cyclide, then the derived surface is the complete parallel of a second cyclide.

Consider next the case where the surface is enveloped by $\infty^{2}$ spheres belonging to an orthogonal complex, $\mathrm{i}$. e., is a surface of the type discussed by Moutard. We are led to the following :

A non-oriented surface enveloped by $\infty^{2}$ spheres of an orthogonal complex $I$, by the inversion $\left(A_{0}\right)$ which carries $(I)$ over into the complex of all points, inverts into a non-oriented surface which is also enveloped by $\infty^{2}$ spheres belonging to $(I)$. The distinction between primitive and derived disappears. The loci of centers are polar reciprocals under polar reciprocation in the fundamental sphere. The intersection of each surface with the fundamental sphere is a focal line of the other surface.* Theorems 4 and 7 still hold.

In particular, if one surface is a cyclide so is the other, either having the above relations to each of a system of five cyclides.

Finally, let the surface be enveloped by $\infty^{2}$ spheres belonging to a plane complex $E$.

\footnotetext{
* It is understood that the inversions involved are related as follows: $F(A) F ; f\left(A_{0}\right) F$; $(A)=\left(A_{0} \Omega A_{0}\right) ;\left(A D^{\prime}\right)=\left(D^{\prime} A\right) ;\left(D^{\prime}\right)=\left(A_{0} I^{\prime} A_{0}\right) ;(A)=(D I D) ; F^{\prime}(D) F$.

* In formulas $(7), \& 5, d_{0}=-r_{0} \sqrt{-1}$.
} 
Theorems $1,3,4,7$ of $\S 4$ are true in this case.* The remaining theorems are replaced by the following:

The oriented surface $F$ derived by inversion in a plane complex from a primitive surface $f$ of order $m$, class $n$, and order of non-singular minimum developable $2 N$, is of order $2(m+N)$, class $2 n$, passing $n$ times through the imaginary circle. The locus of centers of $F^{\prime}$ is derived from $f$ by changing its ordinates relative to the fundamental plane in a certain constant ratio.

The case in which the primitive is a central quadric is discussed at length in the paper by the author already referred to. An incomplete analytical discussion is given by Darboux, loc. cit., vol. II, p. 335 .

The theorems given in section 5 are true in this special case. The equations of the transformation as affecting surface elements may be derived from the general case by a simple transformation with passage to a limit.

\section{§9. Generalization for space of $n$ dimensions, in particular, for $n=2$.}

It is obvious that the discussion and results developed in the preceding pages for space of three dimensions are extended to space of $n$ dimensions by certain trivial verbal and literal changes and additions. Passing over questions of this character, we may turn our attention to the problem in the plane, in which case the general theorems take the following form :

An oriented curve $C$ enveloped by $\infty^{1}$ oriented circles belonging to a linear circular complex $A$ may always be derived by a general inversion $\left(A_{0}\right)$ from $a$ primitive curve regarded as a point locus. The order and class of $C$ are equal, and it intersects the line at infinity only in the circular points. Hence all the foci of $C$ are singular, and all its asymptotes are isotropic. The points of intersection of the primitive curve and fundamental circle are nodes on $C$. $A$ circle orthogonal at once to the fundamental circle and to the primitive at the point $P$ intersects the curve $C$ also orthogonally in the two points of contact of $C$ and the circle into which $P$ inverts under $\left(A_{0}\right)$. Certain tangents (equal in number to the nodes) to a circle concentric with the fundamental circle are bitangents of $C$.

If the primitive is of order $m$, class $n$, passing $r$ times through each circular point, the derived curve $C$ is of order and class $2(2 m+n-4 r)$, having at each circular point a multiple point of order $2 m+n-4 r$. In addition, $C$ has $2(m-r)$ nodes on the fundamental circle, and $2(m-r)$ bitangents touching a concentric circle. The centers of the $\infty^{1}$ circles enveloping $C$ lie upon a curve of order $2(m-r)$, class $2 m+n-4 r$, having at the center of $\left(A_{0}\right)$ a multiple point of order $m-2 r$. The locus of centers $C_{0}$ and the primitive curve have equal deficiencies. The curves $C_{0}$ and $C$ have the same foci.

\footnotetext{
* The complex $E$ transforms into the complex of all points by inversion in a plane complex. Cf. Smitr, loc. cit., for further details.
} 
The two continuous systems each of $\infty^{1}$ oriented circles osculating the primitive invert in $\left(A_{0}\right)$ into the osculating circles of the curve $C$. Apart from the nodes, $C$ intersects the fundamental circle in $2(n-2 r)$ points, at which the osculating circles have four-point contact.

As an application consider the case when the primitive curve $c$ is a bicircular quartic, and, therefore, of order 4, class 8, passing twice through each circular point. Such a curve is sibi-reciprocal under four commutative inversions and also under $(\Omega)$. One has the following results :

The curve $C$ is of order and class 16, passes 8 times through each circular point, and is sibi-reciprocal under five commutative inversions $\left(A_{i}\right) . \quad B y$ inversions concentric with the $\left(A_{i}\right) C$ inverts into 5 bicircular quartics $c_{i}$. The curve $C$ is enveloped in five ways by $\infty^{1}$ oriented circles belonging to an $A_{i}$ whose centers lie upon a quartic curve of deficiency unity. These five quartic curves are confocal, their foci being the foci of $C$. There are 20 double points on $C$, four each on a fundamental circle, the points of intersection namely of $c_{i}$ and that circle. Also $C$ is touched by 20 double tangents, arranged in five sets of four each, touching circles concentric with the fundamental circles.

The curve $C$ just discussed is well known as the curve of singularities of the general quadratic circular complex. Its equation contains 12 essential parameters, to be accounted for by the dependence of a bicircular quartic upon 8 and of a general inversion upon 4 . The properties just given are believed to be new.*

SheFfield Scientific Schoor,

New Hayen, Conn.,

June, 1900.

* Cf. again SNyder, Bulletin of the American Mathematical Society, May, 1900. 